\title{
Multi-dimensional study on the treatment of nonspecific neck pain with ultrasound-guided trigger point injection at the myofascial trigger point of upper trapezius muscles
}

\section{Lu Long}

the Second Affiliated Hospital of Chongqing Medical University

Nadia Anwar

the Second Affiliated Hospital of Chongqing Medical University

Shuangyu Li

the Second Affiliated Hospital of Chongqing Medical University

\section{Meng Fan}

Weinan Central Hospital

\section{Li Zhou}

the Second Affiliated Hospital of Chongqing Medical University

\section{Qianwen Zang}

the Second Affiliated Hospital of Chongqing Medical University

\section{Yongyong Li}

the Second Affiliated Hospital of Chongqing Medical University

\section{Sanrong Wang ( $\sim 303953 @$ @ospital.cqmu.edu.cn )}

the Second Affiliated Hospital of Chongqing Medical University

\section{Research Article}

Keywords: nonspecific neck pain, myofascial trigger point, surface electromyography, ultrasonic elastic imaging, infrared thermal imaging

Posted Date: February 4th, 2022

DOI: https://doi.org/10.21203/rs.3.rs-1122835/v1

License: (9) (1) This work is licensed under a Creative Commons Attribution 4.0 International License. Read Full License 


\section{Abstract}

Background: Nonspecific neck pain (NNP) is a common type of neck pain that causes serious health problems and is closely related to myofascial pain syndrome (MPS). Here, we compare the effectiveness of ultrasound-guided trigger point injection therapy (TPI) and conventional physical therapy treatment in patients with NNP in multiple dimensions.

Methods: Thirty-eight patients with NNP were randomly assigned to the control group and ultrasoundguided TPI group, a single dose of $3 \mathrm{ml}$ of $0.5 \%$ lidocaine was injected into each trigger points in all affected muscles under ultrasound guidance once a week for three weeks. The controls received conventional physical therapy treatment every other day for a total of three weeks. The visual analog scale (VAS) score, neck disability index (NDI), surface electromyography (sEMG), sonoelastography and infrared thermography characteristics were used to measured the effectiveness one week and four weeks after the whole treatment.

Results: Compared with the control group, the TPI group showed improved pain and functional disability both at one-week and four-week assessment $(P<0.01)$; and the TPI group showed improved electrical activity $(P<0.05)$, elastic stiffness and skin temperature at the four-week assessment $(P<0.01)$.

Conclusions: Ultrasound-guided TPI on upper trapezius muscle can alleviate the pain, improves dysfunction for NNP than conventional physical therapy, and the multiple evaluation methods in treatment is more conducive to explore the underlying mechanism of NNP.

Trial registration: The present study has been retrospectively registered on Chinese Clinical Trial Registry website (ChiCTR2100051009), Registered 10 September 2021.

\section{Background}

Neck pain is the main or secondary symptom of diseases such as headache, temporomandibular joint disorder and inflammatory joint disease. The current research on neck pain mainly focuses on structural changes in the neck caused by acute and chronic injury of bones, joints, nerves or blood vessels[1, 2]. However, in clinical practice, a large number of patients with neck pain show symptoms and signs in muscles, ligaments, tendons, and joint capsules, that interfere with daily life activities without specific pathological manifestations, characteristic signs or symptoms. Therefore, patients with this type of neck pain are considered to have nonspecific neck pain (NNP) $[2,3]$.

myofascial pain syndrome (MPS) is regional pain that arises from hyperirritable spots located within taut bands of skeletal muscle, called myofascial trigger points (MTrPs)[4]. MTrPs are discrete lesions in the muscle that contract excessively during palpation. They can be either active or latent; active MTrPs cause spontaneous discomfort, pain, and motor or autonomic symptoms during palpation, whereas latent MTrPs do not cause painful symptoms [5,6]. Myofascial pain is a common type of neck and shoulder pain, the pain can radiate to the upper limbs or head, and the neck and shoulders can have cords or 
nodules that are tender on palpation. In addition, MTrPs often appear in the upper trapezius muscle, and are a common cause of NNP and cervical spine dysfunction. It has been reported that approximately $85 \%$ of patients with MPS have trigger points in the trapezius muscle[7]. Numerous studies have linked MtrPs in the trapezius muscle to NNP in the past few years $[8,9]$.

With technological advancements in imaging modalities, attempts have been made to use imaging techniques to diagnose diseases and evaluate curative effects in recent years [10, 11]. Musculoskeletal ultrasound has been widely used to detect structural changes in tissues and joints. It can be used to assess not only therapeutic effects and structural imaging changes during treatment, but also structural changes of damaged tissues or joints before and after treatment[12, 13]. Elastography can quantify the elastic properties of tissues, and shear wave elastography (SWE) is ultrasonic elastography technology that has immerged in recent years $[14,15]$. Surface electromyography (sEMG) detects the bioelectrical properties of muscle during peripheral nerve stimulation, contraction or rests, and reflects the state of muscle tissue. Infrared thermal imaging (ITI) technology is a kind of functional imaging that uses the principle of infrared radiography to study the temperature distribution of the human body; it reflects the physiological changes and metabolic processes in the human body[16, 17].

Trigger point injection (TPI) is a simple method that involves the injection of drugs into nodules formed by trigger points or muscle tension; it is usually effective and is widely used to relieve myofascial pain. Previous studies demonstrated that TPI improves quality of life in patients with MPS by inactivating trigger points. At present, TPI therapy has been widely used for MPS in clinical practice due to its rapid analgesic effect and easy application[18-20].

In the present study, we aimed to combine physical examination and clinical two-dimensional musculoskeletal ultrasound to examine myofascial activity and potential trigger points to achieve precise positioning of MTrPs. We used objective, reliable outcome measurement tools (VAS, NDI, sEMG, US elasticity imaging and ITI) to evaluate the effectiveness TPI therapy and thoroughly explain their effects and mechanisms in multiple dimensions for NNP with MTrPs in upper trapezius. This study aims to provide new theoretical bases, targets and strategies for the prevention and treatment of NNP with upper trapezius MTrPs.

\section{Materials And Methods}

Participants: This study recruited 38 patients with NNP ranging in age from 20 to 60 years who had been treated on an inpatient or outpatient basis at our institution. The details and procedures of the study were explained to the study participants, who all signed informed consent forms before being included in the

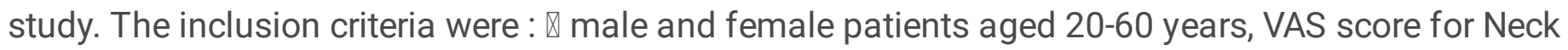
skeletal muscle pain was greater than or equal to 3 points, $\Downarrow$ chronic neck and surrounding tissue pain for more than 3 months, $\nabla$ patient had a diagnosis of at least one active trigger point on the neck, $\nabla$ reduced neck and shoulder range of motion due to pain, and $\otimes$ increased pain density caused by stress or lack of sleep. To improve validity, study subjects were included when all described conditions were satisfied. 
In addition, NNP patients were excluded if they $₫$ were younger than 20 years old or older than 60 years old, regardless of sex, $\nabla$ were diagnosed with temporomandibular arthritis or fibromyalgia, 囚had uncontrollable pain or other discomfort during treatment, $₫$ had excessive muscle tension or mental anxiety during injection treatment or severe swelling and bleeding after injection treatment, $\otimes$ had acute pain in spine (< three months) ,radiculopathy and other spine related conditions.

Patients were randomly assigned into the control group $(n=18)$ or ultrasound-guided TPI group $(n=20)$ by using computer-generated randomization allocation software, Sex was not taken into account during the randomization. The allocation procedure of the participants is presented in the Flow Chart.

\section{Interventions:}

Patients with spine, nerve roots or other spinal-related diseases were ruled out by using magnetic resonance imaging (MRI). The therapist used a combination of physical examination and ultrasound examination to locate and mark the MTrPs of the upper trapezius muscle (Figure 1). All the therapists, evaluators and statisticians were blinded to the participants data.

For participants in the TPI group, a single dose of $3 \mathrm{ml}$ of $0.5 \%$ lidocaine was injected into each trigger points in all affected muscles under ultrasound guidance once a week for 3 weeks, Patients were asked to sit in a straight, upright position throughout the treatment. The injection was administered with a 25gauge, 1.25-inch long needle with the tip perpendicular to the skin, under ultrasound guidance, a needle was inserted into the upper trapezius muscle until the positions of MTrPs were accurately determined. After the injection, the needle was moved back and forth. The same point was needled repeatedly, and the tip of the needle was withdrawn from the muscle[21].

The participants in the control group received conventional physical therapy treatment (heat therapy and exercise). Heat therapy was applied directly over the trapezius muscle of participants for 15 minutes. Then, they performed exercises under the supervision and assistance of an experienced physiotherapist. Each exercise was repeated 10 times for 5-10 s. The treatment was repeated every other day for a total of three weeks[22].

\section{Outcome measurements:}

The visual analog scale (VAS) is a sensitive and comparable method used to measure general pain intensity. The specific method is as follows: a $10 \mathrm{~cm}$ horizontal line is drawn on paper, one end of the horizontal line is 0 , indicating no pain, the other end is 10 , indicating severe pain, and the middle part indicates varying degrees of pain. The participant is asked to draw a mark on the horizontal line according to their self-perceived degree of pain[23].

The Neck Disability Index (NDI) was used to evaluate cervical spine function. The evaluation form included a total of 10 questions concerning neck pain-related symptoms (pain intensity, headache, concentration and sleep) and activities of daily living (personal care, lifting heavy objects, reading, working, driving and entertaining). The patient answered truthfully according to his own situation. The 
higher the score, the more severe the dysfunction is: with zero indicating no disability and five indicating complete disability $(0=$ no disability, $5=$ complete disability) [24].

A Sonosite M-Turbo US system (FUJIFILM Sonosite Inc., Bothell, WA, USA) with a transducer cover and sterile coupling gel was used to locate the upper trapezius trigger points. Scanning was performed using a 13-6 MHz linear transducer. Normally, there are some grayscale degree and pattern changes in areas with MTrPs that appear as focal hypoechoic and heterogeneous echotexture regions. Therefore, we used musculoskeletal US to locate, guide and administer TPI for NNP, and evaluated the effect of myofascial structural changes before and after treatment[25,26].

sEMG analysis was performed with a device (Shanghai Nuocheng Electric Co., LTD) on the upper part of the trapezius muscle bilaterally. The motor unit activity in EMG recordings ranges from $70-90 \mathrm{~Hz}$, the time base for recording was $1000 \mathrm{~ms}$, the scanning speed was $3 \mathrm{~s} / \mathrm{D}$, and the amplification was $80 \mu \mathrm{V} / \mathrm{D}$ during maximal contraction of muscle. A pair of the standard bipolar electrodes was placed on the surface of the skin over the muscle belly. The SEMG recordings were performed during maximal voluntary contraction of-the trapezius muscle for $5 \mathrm{~s}$ and repeated for 3 times to measure the amplitude [27].

The soft tissue stiffness of each point was assessed by sonoelastography. An musculoskeletal ultrasound system (SuperSonic Imagine Aixplorer, France) with an SL15-4 linear transducer and frequency of 4-15 MHz was used to perform resting mode sonoelastography in the sitting position. To perform a comprehensively inspect the muscles at the mark, the detection depth was set to $2 \sim 3 \mathrm{~cm}[28]$.

Infrared thermography (TMT-9000P, Hangzhou Xinhan Photoelectric Technology Co., LTD.) was used to measure skin temperature changes over the upper trapezius muscle in our special infrared shielding room. The patient was instructed to sit and rest for 5-10 minutes before the test, and then positioned 2.5 $\mathrm{m}$ from the infrared camera for ITI according to the prescribed standard position. The infrared thermal images taken were analyzed by software, and the difference in body surface temperature was distinguished by the difference in color scale[29].

\section{Statistical analyses:}

The total sample size was calculated to be 40 (20 in each group) with a $10 \%$ acceptable drop-out rate by using G-POWER software. The descriptive statistics are shown as the calculated mean and standard deviation. One-way ANOVA was used to perform intergroup comparisons of the baseline data. T-tests were used to compare the effect before and after treatment in terms of VAS score, NDI, sonoelastography, EMG and infrared thermography data in the two groups. Similarly, T-tests were used to compare results between the two groups. All statistical analyses were performed with SPSS (version 23, IBM Corp., Armonk, NY, USA). All tests of statistical significance were interpreted with a criterion of $p<0.05$.

\section{Results}


There was no significant difference in age, sex, occupation, height, weight, BMI, or the presence of NNP with upper trapezius MTrPs (Table 1, P>0.05). No side effects or complications were seen in either group during the study. Significant changes could be seen between the two groups (TPI group vs. the control group) both at one week and four weeks after the last time treatment.

Table 1 Demographic characteristics of the participants

\begin{tabular}{|llll|}
\hline Variables & $\begin{array}{l}\text { Control group }(\mathrm{n}=16) \\
(\mathrm{mean} \pm \text { SD })\end{array}$ & $\begin{array}{l}\text { TPI group }(\mathrm{n}=18) \\
(\text { mean } \pm \text { SD })\end{array}$ & p-value \\
\hline Age & $41.07 \pm 11.63$ & $45.44 \pm 10.09$ & 0.27 \\
\hline Sex & $1.69 \pm 0.48$ & $1.66 \pm 0.48$ & 0.88 \\
Occupation & $1.84 \pm 0.68$ & $1.72 \pm 0.66$ & 0.61 \\
\hline Height $(\mathrm{cm})$ & $159.7 \pm 6.9$ & $162.5 \pm 6.7$ & 027 \\
\hline Weight $(\mathrm{kg})$ & $55.9 \pm 9.6$ & $59.6 \pm 8.1$ & 0.25 \\
\hline BMI $\left(\mathrm{kg} / \mathrm{m}^{2}\right.$ & $21.9 \pm 3.7$ & $22.6 \pm 3.0$ & 0.57 \\
\hline
\end{tabular}

The VAS scores decreased significantly in both two groups at the one-week and four-week follow up compared with those at baseline (Figure $2 A, P<0.01$ ). There was no significant difference in VAS scores at baseline between the two groups (Figure $2 \mathrm{~A}, \mathrm{P}>0.05$ ). Although the patients in the control group also benefited from conventional physical treatment, those in the TPI group benefited more in terms of pain relief, as observed at all time points with VAS scoring (Figure $2 A, P<0.01$ ).

In terms of functional disability, the NDI score in the control group significantly decreased one week after treatment compared with baseline, but the NDI did not continue to decrease four weeks after treatment (Figure 2B, $\mathrm{P}<0.01$ ). However, the TPI group showed a steady decrease in NDI scores until four weeks after treatment (Figure $2 B, P<0.01$ ). There was no significant difference in NDI score at baseline between the two groups (Figure $2 B, P>0.05$ ). A significant difference was found in NDI scores between the two groups (Figure $2 \mathrm{~B}, \mathrm{P}>0.05$ ).

In addition, the control group showed a significant difference in EMG results at one week after intervention compared with baseline (Figure $2 \mathrm{C}, \mathrm{P}<0.05)$ ). There was no significant difference in electrical activity four weeks after treatment (Figure $2 \mathrm{C}, \mathrm{P}>0.05$ ). In contrast, the TPI group showed a significant difference in electrical activity at the four-week assessment (Figure $2 \mathrm{C}, \mathrm{P}<0.05$ ). There was no significant difference in EMG results at baseline and one week after intervention between the two groups (Figure 2C, $P>0.05)$. However, the EMG results were significantly different between the two groups at the four-week assessment (Figure $2 \mathrm{C}, \mathrm{P}<0.05$ ). 
However, no significant difference in sonoelastography results was found in the control group (Figure 3A$B, P>0.05)$. In contrast, the stiffness of MTrPs was significantly decreased one week after treatment and decreased more at the four-week assessment in the TPI group (Figure 3A-B, $P<0.01$ ). There was no significant difference in sonoelastography results at baseline and one week after treatment between the two groups (Figure $3 C, P>0.05$ ). The sonoelastography results in the TPI group were lower than that in the control group at the four-week assessment (Figure 3C, $\mathrm{P}<0.01$ ).

Infrared thermography showed no significant improvement over time in the control group (Figure 4A-B, $P>0.05)$. The skin temperature in the TPI group significantly decreased at the one-week and four-week assessments; moreover, the improvement changes were significantly higher at the four-week assessment than that at the one-week assessment (Figure 4A-B, $P<0.01$ ). There was no significant difference in infrared thermography results at baseline and one week after treatment between the two groups (Figure $4 C, P>0.05)$. However, there is a significant difference between the two groups four weeks after treatment (Figure $4 \mathrm{C}, \mathrm{P}<0.01$ ).

\section{Discussion}

Due to the changes in social life, working modes and the application of a large number of electronic components and devices, an increasing number of people suffer from NNP[30]. In 2017, The Lancet reported that the year-to-year life loss with disability (YLD) for neck pain increased by $21.9 \%$ from 2006 to $2016[31,32]$, which urgently needs to be addressed. The question addressed by the present study was whether -ultrasound guided TPI therapy has a positive effect on NNP with MTrPs in the upper trapezius muscle; and whether it has advantages in clinical application compared with traditional physiotherapy. Our study found that the ultrasound guided TPI therapy was effective for NNP with MTrPs in the upper trapezius muscle in improving pain intensity, functional disability, electrical activity, elastic stiffness and skin temperature changes.

The diagnosis of MPS, which manifests with one or more active MTrPs, is usually based on the patient's subjective symptoms and the presence of active MTrPs. However, due to the subjective and empirical nature of physical examination, it is difficult to find potential and deep tissue trigger points, and its reliability is controversial. Therefore, it is particularly important to seek an objective and accurate method for identifying the trigger points[33,34]. In recent years, a large number of studies have shown that MTrPs are mostly oval hypoechoic areas on ultrasound images, and there are very few case reports that MTrPs are hyperechoic[35, 36]. Cojocaru M C et al.[37] simultaneously applied US imaging, ITI and clinical palpation techniques to locate the MTrPs in 8 patients with lumbar pain. They found that these three methods were basically consistent in diagnosing the location of MTrPs. Kang JJ et al.[18] compared the feasibility of ultrasound guided MTrP injection with that of blind injection following the use of SWE for the assessing stiffness at the MTrPs in patients with trapezius MPS, they found that ultrasound guided TPI is a more useful modality than blind injection in patients with MPS. The use of ultrasound guided TPI 
addresses the limitations of palpation and blind injection; it can not only help identify and treat MTrPs in deep muscles, but also avoid damage to nerves, blood vessels and organs, and improve the accuracy and effectiveness of MTrP injection.

TPI therapy has been used for decades, and it has obtained satisfactory results in clinical treatment of MPS. Our research results are consistent with many clinical studies, showing that ultrasound guided TPI therapy improves functional recovery and reduces pain associated with NNP. Raeissadat SA et al.[38] compared the efficacy of ozone injection (OI) lidocaine injection (LI) and dry needling (DN) in MPS patients. The results showed that all three methods were significantly effective in MPS treatment during short-term follow-up; however, the OI and LI groups had slightly better results than the DN group, with no remarkable benefit between OI and LI. Because ultrasound guided local anesthetic injections are superior to nonspecific anti-inflammatory drugs (NSAIDs) in terms of pain outcomes and fewer adverse events were found in a systematic review[39], we chose ultrasound guided injection of $5 \%$ lidocaine in the present study, and no adverse events occurred over the course of the experiment. In an early study of the treatment of NNP, Yanuck $\mathrm{J}$ et al.[40]evaluated whether TPI with 1\% lidocaine can improve myofascial back and neck pain compared with conventional therapies (standard care). They found that numerical rating score (NRS) for pain was lower in the TPI group than the control group after adjustment for initial pain. The VAS scores decreased significantly in the TPI groups at the one-week and four-week assessments compared to baseline in the present study.

The pathophysiological mechanism of trigger point formation in the upper trapezius muscle in patients with NNP is unclear, and muscle overloading is reported to be the key factor. In addition, lack of physical exercise, acute trauma and long periods of poor posture are also linked to the formation of trigger points[41]. Simons et al. [42]argued in their study that long-term micro muscle injury would cause continuous contraction of muscle fibers, which would increase local energy consumption. Meanwhile, contracture of muscle nodes puts pressure on local blood vessels, leading to local microcirculation disturbances and the release of pain-causing substances such as 5-hydroxytryptamine and bradykinin, eventually forming MTrPs. A recent study also found that skin temperatures were higher in areas with MTrPs, which may be related to the rise in skin temperature caused by the heat generated by the tension of muscles with MTrP activation[43]. As demonstrated in our observations, ultrasound guided TPI has an improving effect on neck pain relief, functional disability, electrical activity, elastic stiffness, and skin temperature over upper trapezius MTrPs in NNP. The proposed mechanism mediating this therapeutic effect is that lidocaine, an amide anesthetic drug, can effectively relieve the pain of patients, improve blood circulation, reduce exudation and accelerate the metabolism of local inflammatory substances. At the same time, needling destroys MTrPs, reduces the activity of trigger points, disrupts the original continuous contraction state of muscles, reduces the tension of local muscle tissue, relives the symptoms of neurovascular entrapment, promotes local blood circulation, and releases tissue growth factor, thus stimulating a healing response and facilitating the discharge of metabolic waste and paincausing factors[19, 44]. 
Previous therapeutic assessments of NNP have relied on some subjective evaluation scales. The most commonly used are the VAS and NDI. In contrast to previous studies, the present study is the first to combine multiple objective, reliable outcome measurement tools concerning pain intensity, functional disability and electrical activity to evaluate the short-term curative effect of ultrasound guided TPI therapy on NNP. The present results provide strong evidence to support the effectiveness of ultrasound guided TPI for NNP with trigger points in the upper trapezius muscle, which has great value for clinical application.

\section{Conclusions}

The present study revealed that ultrasound-guided TPI on upper trapezius muscle can alleviate the pain, improves dysfunction for NNP than conventional physical therapy, and the multiple evaluation methods in treatment is more conducive to explore the underlying mechanism of NNP.

\section{Abbreviations}

Nonspecific neck pain (NNP), myofascial pain syndrome (MPS), myofascial trigger points (MTrPs), trigger point injection therapy (TPI), The visual analog scale score(VAS), neck disability index (NDI), surface electromyography (sEMG), shear wave elastography (SWE), Infrared thermal imaging (ITI).

\section{Declarations}

Ethics approval and consent to participate: The trial was conducted according to the principles of the Declaration of Helsinki. Ethical Committee for Research, Second Affiliated Hospital of Chongqing Medical University approved the present study, the present study has been registered on Chinese Clinical Trial Registry website (ChiCTR2100051009). Participants provided written informed consent prior to commencing the trial.

Consent for publication: Not applicable. The manuscript does not contain patient information or details.

Availability of data and materials: The datasets used and/or analysed during the current study are available from the corresponding author upon reasonable request.

Conflicts of interest: The authors declare that they have no competing interests.

Funding: This work was supported by the National Natural Science Foundation of China (NSFC, 81601967) and Chongqing Health Joint Medical Research Project: 2021MSXM144.

Authors' contributions: All the authors contributed to the study concept and design. Lu Long, Nadia Anwar, Shuangyu Li, Meng Fan, Li Zhou, Qianwen Zang and Yongyong Li analyzed and interpreted data on patients with NNP. Wang Sanrong performed the TPI treatment, and Long Lu was the main contributor to the manuscript. All authors read and approved the final manuscript. 
Acknowledgement: We would like to thank the participants for their excellent cooperation.

\section{References}

1. Bogduk N. The anatomy and pathophysiology of neck pain. Physical Medicine and Rehabilitation Clinics of North America. 2003,14(3):455-72.

2. Bogduk N. The anatomy and pathophysiology of neck pain. Physical Medicine and Rehabilitation Clinics of North America. 2011,22(3):367-82, vii.

3. Zaproudina N, Ming Z, Narhi M. Sensory and sympathetic disorders in chronic non-specific neck pain. Funct Neurol. 2015,30(3):165-71.

4. Ware J. Misreport of trigger point diagnosis reliability. The Journal of orthopaedic and sports physical therapy. 2015,45(2):144-5.

5. Kameda $\mathrm{M}$, Tanimae $\mathrm{H}$. Effectiveness of active soft tissue release and trigger point block for the diagnosis and treatment of low back and leg pain of predominantly gluteus medius origin: a report of 115 cases. Journal of physical therapy science. 2019,31(2):141-8.

6. Mense S. Nociception from skeletal muscle in relation to clinical muscle pain. Pain. 1993,54(3):24189.

7. Yildirim M, Öneş K, Gökşenoğlu G. Effectiveness of Ultrasound Therapy on Myofascial Pain Syndrome of the Upper Trapezius: Randomized, Single-Blind, Placebo-Controlled Study. Archives of rheumatology. 2018,33(4):418-23.

8. Cerezo-Téllez E, Torres-Lacomba M, Mayoral-Del Moral O, Sánchez-Sánchez B, Dommerholt J, Gutiérrez-Ortega C. Prevalence of Myofascial Pain Syndrome in Chronic Non-Specific Neck Pain: A Population-Based Cross-Sectional Descriptive Study. Pain medicine (Malden, Mass). 2016,17(12):2369-77.

9. Fernandez-de-las-Penas C, Alonso-Blanco C, Miangolarra JC. Myofascial trigger points in subjects presenting with mechanical neck pain: a blinded, controlled study. Manual therapy. 2007,12(1):29-33.

10. Turo D, Otto P, Shah J, Heimur J, Gebreab T, Zaazhoa M, et al. Ultrasonic characterization of the upper trapezius muscle in patients with chronic neck pain. Ultrasonic imaging. 2013,35(2):173-87.

11. Lin Y, Yu N, Jiang C, Chang S. Characterizing the SEMG patterns with myofascial pain using a multiscale wavelet model through machine learning approaches. Journal of electromyography and kinesiology : official journal of the International Society of Electrophysiological Kinesiology. 2018,41:147-53.

12. Botwin K, Sharma K, Saliba R, Patel B. Ultrasound-guided trigger point injections in the cervicothoracic musculature: a new and unreported technique. Pain physician. 2008,11(6):885-9.

13. Jafari M, Bahrpeyma F, Mokhtari-Dizaji M, Nasiri A. Novel method to measure active myofascial trigger point stiffness using ultrasound imaging. Journal of bodywork and movement therapies. 2018,22(2):374-8. 
14. Sikdar S, Shah J, Gilliams E, Gebreab T, Gerber L. Assessment of myofascial trigger points (MTrPs): a new application of ultrasound imaging and vibration sonoelastography. Annual International Conference of the IEEE Engineering in Medicine and Biology Society IEEE Engineering in Medicine and Biology Society Annual International Conference. 2008,2008:5585-8.

15. Muller CE, Aranha MF, Gaviao MB. Two-dimensional ultrasound and ultrasound elastography imaging of trigger points in women with myofascial pain syndrome treated by acupuncture and electroacupuncture: a double-blinded randomized controlled pilot study. Ultrason Imaging. 2015,37(2):152-67.

16. Ring EF, Ammer K. Infrared thermal imaging in medicine. Physiological measurement. 2012,33(3):R33-46.

17. Ballyns JJ, Turo D, Otto P, Shah JP, Hammond J, Gebreab T, et al. Office-based elastographic technique for quantifying mechanical properties of skeletal muscle. J Ultrasound Med. 2012,31(8):1209-19.

18. Kang J, Kim J, Park S, Paek S, Kim T, Kim D. Feasibility of Ultrasound-Guided Trigger Point Injection in Patients with Myofascial Pain Syndrome. Healthcare (Basel, Switzerland). 2019,7(4).

19. Xie $P$, Qin $B$, Yang F, Yu T, Yu J, Wang J, et al. Lidocaine Injection in the Intramuscular Innervation Zone Can Effectively Treat Chronic Neck Pain Caused by MTrPs in the Trapezius Muscle. Pain physician. 2015,18(5):E815-26.

20. Tang TY, Shyu SG, Kao BC, Wu CH. Ultrasound-Guided 5-in-1 Injection: Trigger Point Injections and Nerve Hydrodissections for Nonspecific Upper Back Pain. American journal of physical medicine \& rehabilitation. 2019,98(6):e55-e6.

21. Ay S, Evcik D, Tur BS. Comparison of injection methods in myofascial pain syndrome: a randomized controlled trial. Clin Rheumatol. 2010,29(1):19-23.

22. Aslan Telci E, Karaduman A. Effects of three different conservative treatments on pain, disability, quality of life, and mood in patients with cervical spondylosis. Rheumatol Int. 2012,32(4):1033-40.

23. Sung Y, Wu J. The Visual Analogue Scale for Rating, Ranking and Paired-Comparison (VAS-RRP): A new technique for psychological measurement. Behavior research methods. 2018,50(4):1694-715.

24. Oliver JD, Goncalves S, Kerezoudis P, Alvi MA, Freedman BA, Nassr A, et al. Comparison of Outcomes for Anterior Cervical Discectomy and Fusion With and Without Anterior Plate Fixation: A Systematic Review and Meta-Analysis. Spine. 2018,43(7):E413-E22.

25. Kumbhare DA, Elzibak AH, Noseworthy MD. Assessment of Myofascial Trigger Points Using Ultrasound. American journal of physical medicine \& rehabilitation. 2016,95(1):72-80.

26. Park CH, Lee YW, Kim YC, Moon JH, Choi JB. Treatment experience of pulsed radiofrequency under ultrasound guided to the trapezius muscle at myofascial pain syndrome -a case report. The Korean journal of pain. 2012,25(1):52-4.

27. Fridlund AJ, Cacioppo JT. Guidelines for human electromyographic research. Psychophysiology. 1986,23(5):567-89. 
28. Calvo-Lobo C, Diez-Vega I, Martínez-Pascual B, Fernández-Martínez S, de la Cueva-Reguera M, Garrosa-Martín G, et al. Tensiomyography, sonoelastography, and mechanosensitivity differences between active, latent, and control low back myofascial trigger points: A cross-sectional study. Medicine. 2017,96(10):e6287.

29. Zaproudina N, Airaksinen O, Narhi M. Are the infrared thermography findings skin temperaturedependent? a study on neck pain patients. Skin research and technology : official journal of International Society for Bioengineering and the Skin. 2013,19(1):e537-44.

30. Wolff R, Clar C, Lerch C, Kleijnen J. [Epidemiology of chronic non-malignant pain in Germany]. Schmerz. 2011,25(1):26-44.

31. Cerezo-Tellez E, Torres-Lacomba M, Mayoral-Del-Moral O, Pacheco-da-Costa S, Prieto-Merino D, Sanchez-Sanchez B. Health related quality of life improvement in chronic non-specific neck pain: secondary analysis from a single blinded, randomized clinical trial. Health and quality of life outcomes. 2018,16(1):207.

32. Global, regional, and national incidence, prevalence, and years lived with disability for 328 diseases and injuries for 195 countries, 1990-2016: a systematic analysis for the Global Burden of Disease Study 2016. Lancet (London, England). 2017,390(10100):1211-59.

33. Lucas N, Macaskill P, Irwig L, Moran R, Bogduk N. Reliability of physical examination for diagnosis of myofascial trigger points: a systematic review of the literature. Clin J Pain. 2009,25(1):80-9.

34. Lew PC LJ, Story I. Inter-therapist reliability in locating latent myofascial trigger points using palpation. Manaul Therapy 1997 2(2):87-90. .

35. Shankar H, Reddy S. Two- and three-dimensional ultrasound imaging to facilitate detection and targeting of taut bands in myofascial pain syndrome. Pain Med. 2012,13(7):971-5.

36. Shankar $\mathrm{H}$, Cummings $\mathrm{C}$. Ultrasound imaging of embedded shrapnel facilitates diagnosis and management of myofascial pain syndrome. Pain practice : the official journal of World Institute of Pain. 2013,13(5):405-8.

37. Cojocaru MC, Cojocaru IM, Voiculescu VM, Cojan-Carlea NA, Dumitru VL, Berteanu M. Trigger pointsultrasound and thermal findings. Medcine Life. 2015,8(3):315-8(1844-3117 (Electronic)).

38. Raeissadat SA, Rayegani SM, Sadeghi F, Rahimi-Dehgolan S. Comparison of ozone and lidocaine injection efficacy vs dry needling in myofascial pain syndrome patients. J Pain Res. 2018,11(11787090 (Print)):1273-9.

39. Diep D, Chen KJQ, Kumbhare D. Ultrasound-guided interventional procedures for myofascial trigger points: a systematic review. Reg Anesth Pain Med. 2021,46(1):73-80.

40. Yanuck J, Saadat S, Lee JB, Jen M, Chakravarthy B. Pragmatic Randomized Controlled Pilot Trial on Trigger Point Injections With 1\% Lidocaine Versus Conventional Approaches for Myofascial Pain in the Emergency Department. The Journal of emergency medicine. 2020,59(3):364-70.

41. Kumbhare D, Singh D, Rathbone HA, Gunn M, Grosman-Rimon L, Vadasz B, et al. Ultrasound-Guided Interventional Procedures: Myofascial Trigger Points With Structured Literature Review. Reg Anesth Pain Med. 2017,42(3):407-12. 
42. Simons DG, Mense S. [Diagnosis and therapy of myofascial trigger points]. Schmerz. 2003,17(6):419-24.

43. Yassin M, Talebian S, Ebrahimi Takamjani I, Maroufi N, Ahmadi A, Sarrafzadeh J, et al. The effects of arm movement on reaction time in patients with latent and active upper trapezius myofascial trigger point. Medical journal of the Islamic Republic of Iran. 2015,29:295.

44. Kamali F, Sinaei E, Morovati M. Comparison of Upper Trapezius and Infraspinatus Myofascial Trigger Point Therapy by Dry Needling in Overhead Athletes With Unilateral Shoulder Impingement Syndrome. Journal of sport rehabilitation. 2019,28(3):243-9.

\section{Supplementary}

Flow chart is available in Supplemental Files section.

\section{Figures}

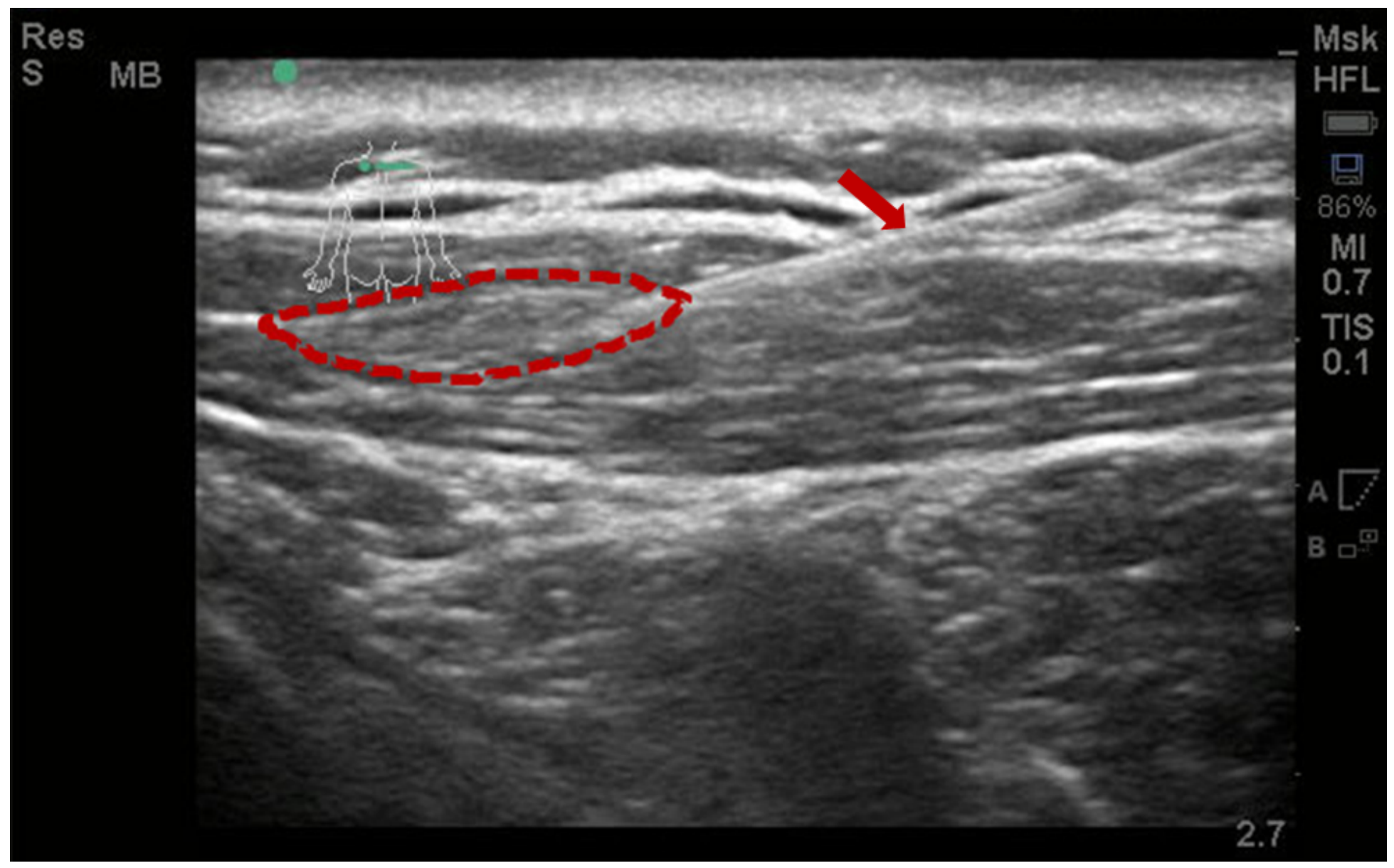

\section{Figure 1}

A typical trigger point can be observed in the left neck, as shown in the dotted-line mark, white capsule can be seen around, and the ultrasound performance shows mixed echo area, and the trigger point injection therapy under ultrasound-guided, the needle as shown in the arrow mark. 
A
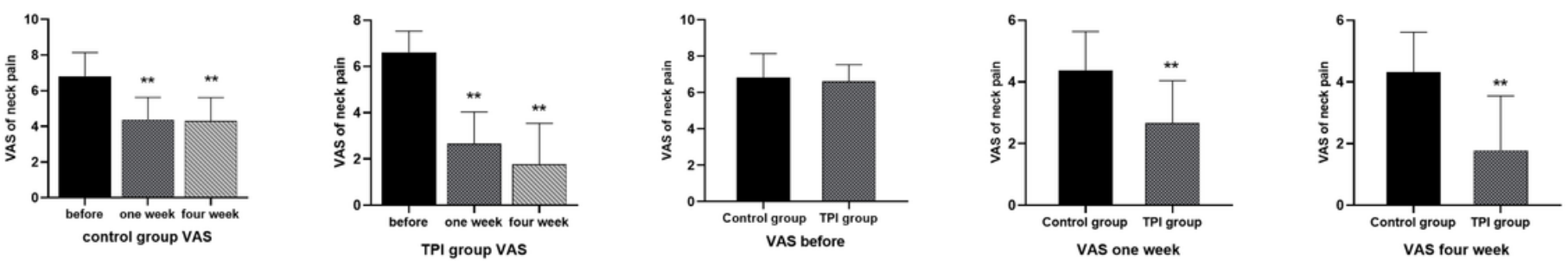

B
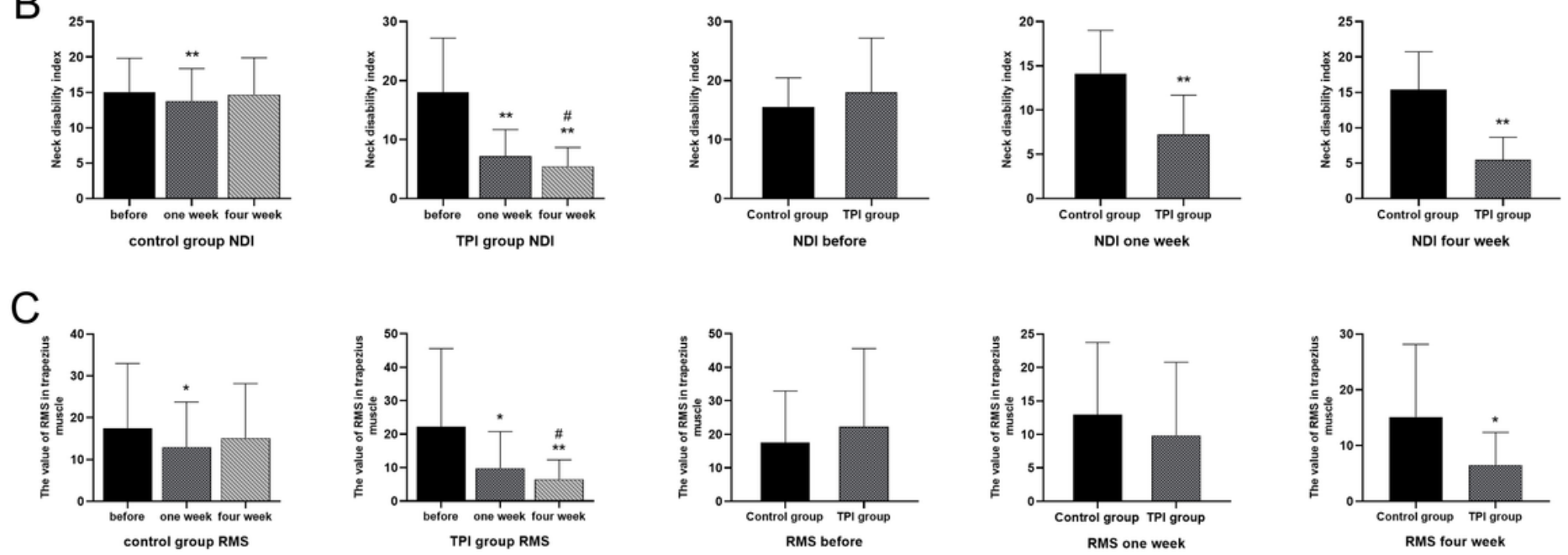

Figure 2

A: VAS of neck pain in each group and VAS of neck pain between groups (mean $\pm S D$ ) at before, one week and four week, B: NDI in each group and NDI between groups (mean \pm SD) at before, one week and four week, C: RMS in each group and RMS between groups (mean \pm SD) at before冈one week and four week, ${ }^{*} p<0.05, \# p<0.05, * * p<0.01, \# \# p<0.01$. 

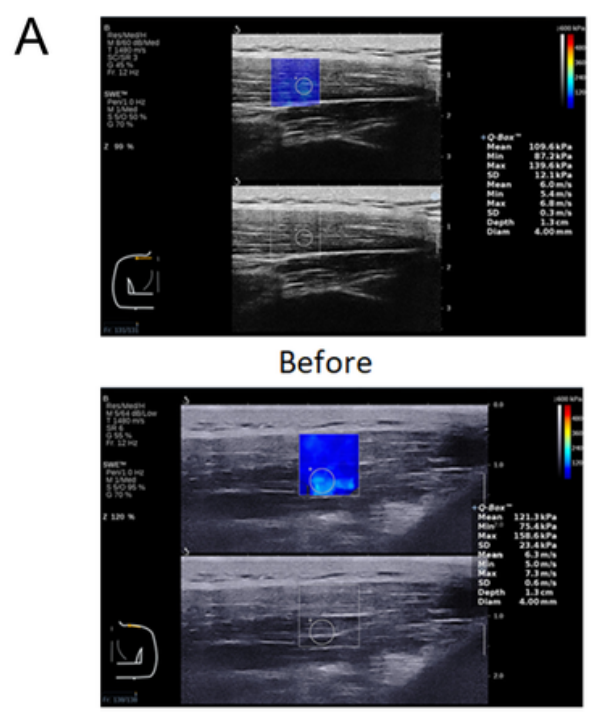

Before
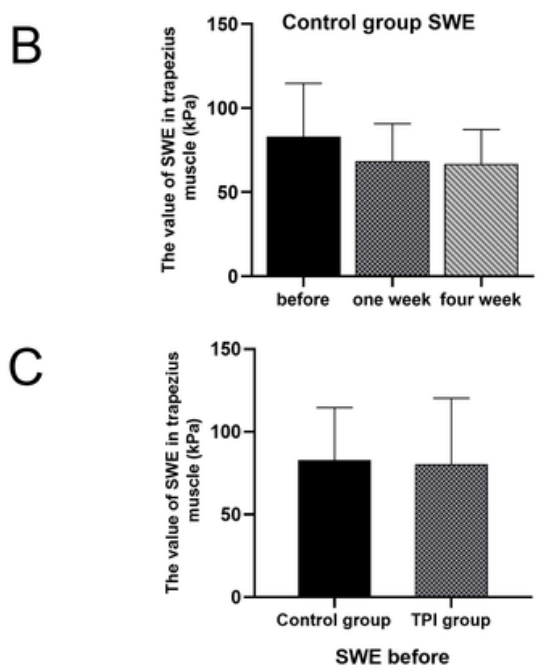

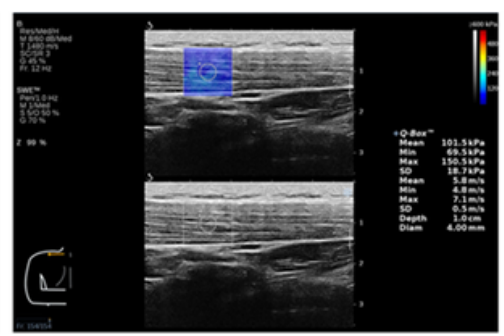

One week

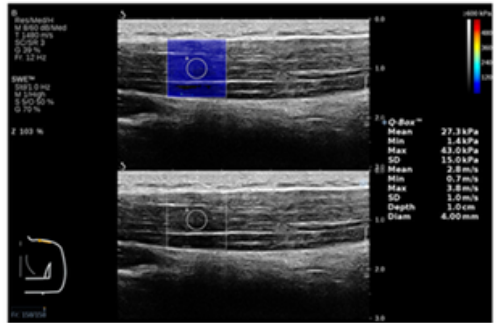

One week
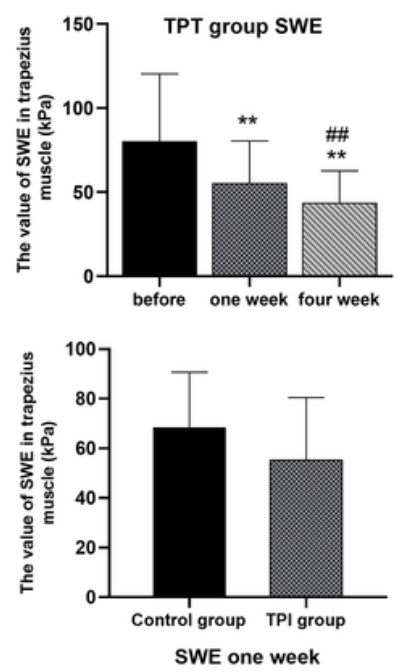

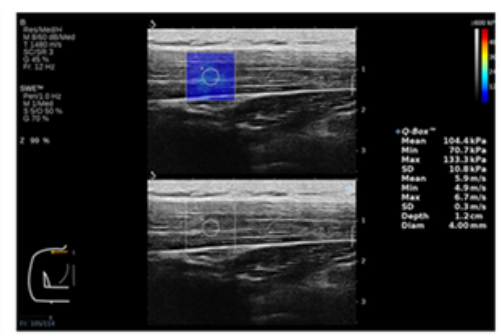

Control group

Four week

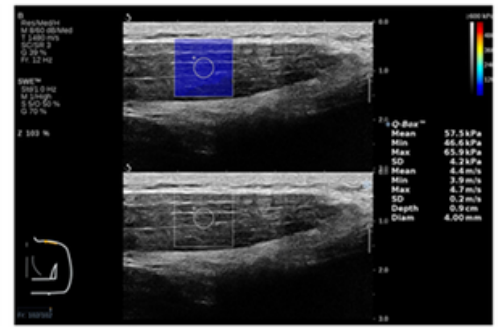

Four week

Injection group

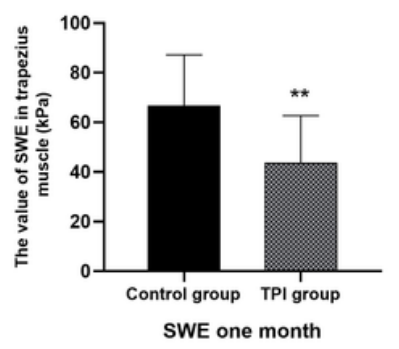

Figure 3

A: Representative measurement of SWE value at the trapezius trigger point in patients with cervical and shoulder myofascial pain syndrome in control group (male, 43 years old, with repeated neck pain for more than three years), injection group (female, 35 years old, with repeated neck pain for more than five years), B: The value of SWE in trapezius muscle $(\mathrm{kPa})$ in each group (mean $\pm \mathrm{SD}$ ) at before, one week and four week time, $\mathrm{C}$ : The value of SWE in trapezius muscle (kPa) between groups (mean \pm SD) at before, one week and four week, ${ }^{*} p<0.05, \# p<0.05,{ }^{*} \mathrm{p}<0.01, \# \# p<0.01$. 
A

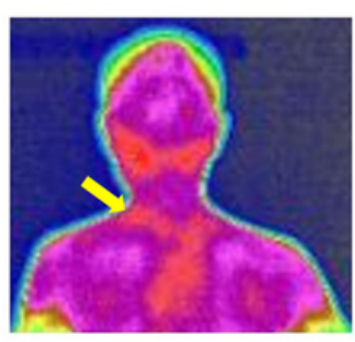

Before

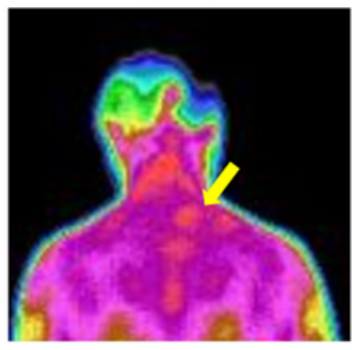

Before

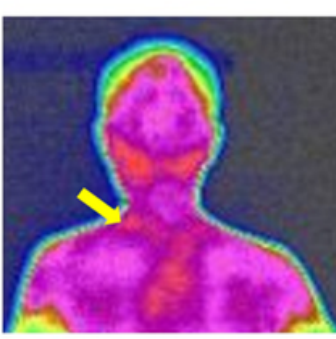

One week

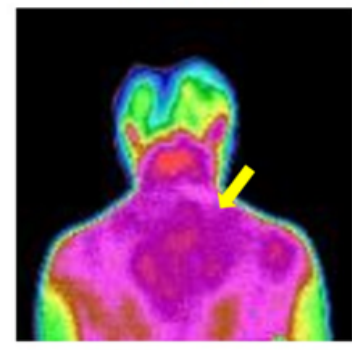

One week

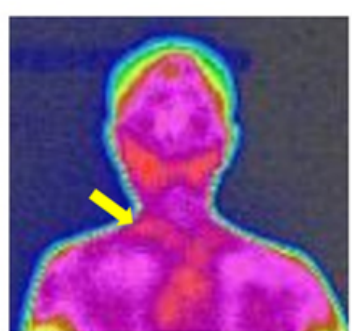

Four week

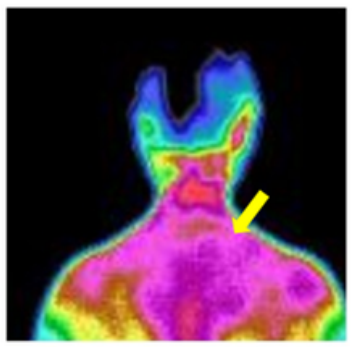

Four week
B

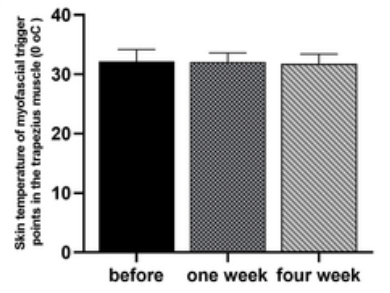

infraredthermograpy of Control group

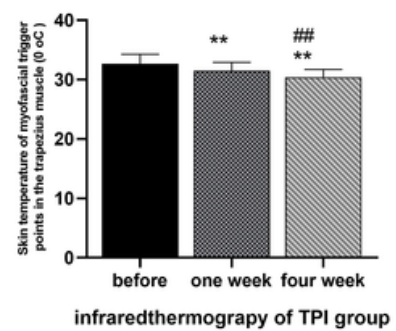

C

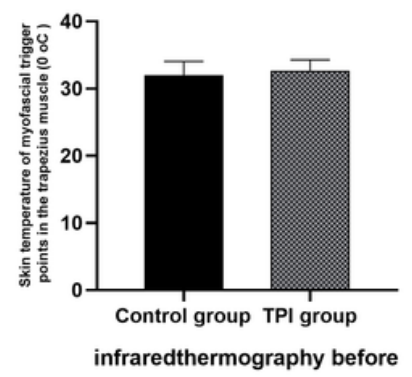

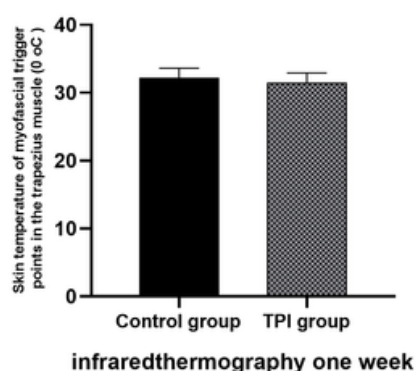

Control group

Injection group

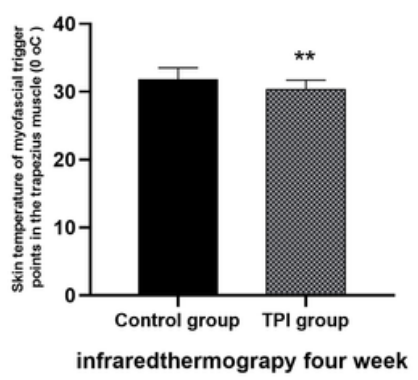

Figure 4

A: Representative measurement of infraredthermography at the trapezius trigger point, as shown in the arrow mark, in patients with cervical and shoulder myofascial pain syndrome in control group (male, 43 years old, with repeated neck pain for more than three years), injection group (female, 35 years old, with repeated neck pain for more than five years). B: Skin temperature of myofascial trigger points in the trapezius muscle $\left({ }^{\circ} \mathrm{C}\right)$ in each group at before, one week and four week time, C: Skin temperature of myofascial trigger points in the trapezius muscle $\left({ }^{\circ} \mathrm{C}\right.$ ) between groups (mean $\pm \mathrm{SD}$ ) at before, one week and four week, ${ }^{*} p<0.05, \# p<0.05,{ }^{*} \mathrm{p}<0.01, \# \# p<0.01$.

\section{Supplementary Files}

This is a list of supplementary files associated with this preprint. Click to download.

- floatimage1.jpeg 